\title{
Traduire
}

Une eutre perspective sur $r$ tatadciction

Revue française de la traduction

$230 \mid 2014$

À la croisée du texte et de l'image

\section{TAPATOUCOMPRIX : le nez de Cléopâtre ou le pouce d'Astérix?}

Jean-François Allain

\section{(2) OpenEdition}

1 Journals

Édition électronique

URL : http://journals.openedition.org/traduire/634

DOI : $10.4000 /$ traduire.634

ISSN : 2272-9992

Éditeur

Société française des traducteurs

Édition imprimée

Date de publication : 15 juin 2014

Pagination : 96-100

ISSN : 0395-773X

\section{Référence électronique}

Jean-François Allain, «TAPATOUCOMPRIX : le nez de Cléopâtre ou le pouce d'Astérix ? », Traduire [En ligne], 230 | 2014, mis en ligne le 15 juin 2016, consulté le 24 septembre 2020. URL : http:// journals.openedition.org/traduire/634 ; DOI : https://doi.org/10.4000/traduire.634 


\section{TAPATOUCOMPRIX : le nez de Cléopâtre ou le pouce d'Astérix?}

\section{Jean-François Allain}

À sept ans, mon tout jeune frère s'asseyait dans les escaliers et récitait, l'air réjoui, des pages entières d'Astérix. À l'époque, je faisais des études de lettres classiques et, fort de ma science, je me demandais ce qu'il pouvait bien " comprendre ". Mais mon père, qui était de la génération de Goscinny et avait étudié le latin chez les Jésuites, m'expliquait de son côté des allusions que je n'avais pas comprises.

Résidant en Grande-Bretagne un peu plus tard, j'ai eu l'occasion de lire l'édition anglaise d'Astérix (" une " édition, car il semble qu'il y en ait eu d'autres depuis) en regrettant naturellement que tant de choses aient été lost in translation. L'album était néanmoins accompagné d'un cahier qui expliquait ce qu'il fallait " comprendre " des multiples jeux sur les mots, les noms propres et les images. Certains m'avaient échappé, d'autres avaient échappé au traducteur ; j'ai donc dressé une petite liste complémentaire que je me promettais d'envoyer à l'éditeur, " pour faire avancer la science ". Cette liste doit sentir le moisi quelque part dans mes cartons d'archives.

Jeux sur les mots, allusions perdues, mais même quand on ne comprend pas l'allusion, on " sent " parfois qu'il y a une allusion, ce qui n'aide pas beaucoup s'il faut la traduire, mais n'est-ce pas déjà amusant? Sur les images, j'étais moins fort. Mon éducation ne me permettait pas de saisir tous les clins d'œil à la culture visuelle de Spirou, Pilote et autres grands noms de la bande dessinée.

À propos d'image et par ce long détour, j'en viens à ce qui motive ma chronique : les actes d'un colloque consacré à Astérix, à la Sorbonne, où l'on apprend par exemple que la signification du pouce levé (pour dire "pouce, on arrête! ") n'est pas comprise en ce sens en Hispanie(1). Et on se dit soudain " Bon sang mais c'est bien sûr ! " (en se frappant le front), même si l'on savait depuis Pascal qu'erreur au-delà des Pyrénées, vérité en deçà (ou vice-versa). Au moins, le geste n'est pas compris, car il est des pays où il serait compris tout autrement et pourrait nous attirer des ennuis.

(1) José Yuste Frias, "Traduire l'image dans les albums d'Astérix ", in Bertrand Richet (éd.), Le Tour du monde d’Astérix, Paris, Presses Sorbonne nouvelle, 2011, p. 263. 
Cela ressemble à une anecdote, mais ce n'en est pas une. C'est une illustration, un exemple représentatif de toute une "problématique de la traduction".

L'auteur, José Yuste Frias, nous rappelle donc opportunément que l'image n'a pas encore la place qu'elle mérite dans les études sur la traduction et il évoque, entre autres, les risques de contre-sens dans les publicités, avec, dans ce cas, des conséquences autrement plus graves pour une entreprise, en termes d'argent ou de réputation.

Après, naturellement, il faut pouvoir expliquer tout ça en mieux, parler de "localisation spatiotemporelle ", employer quelques grands mots et l'on comprend alors que

le traducteur ne traduit jamais des langues ni des mots mais toujours les imaginaires véhiculés non seulement par les images mentales implicites dans le texte, mais aussi par les images matérialisées dans le péritexte iconique, qu'il soit fixe ou animé (p. 255-256),

et on se redit "Bon sang mais c'est bien sûr ! " (en se frappant à nouveau le front).

J'ai l'air ironique, mais c'est pure jalousie. Si je n'avais pas eu toute ma vie autant de traductions à boucler dans les temps, j'aurais aimé être traductologue.

Évidemment, cette publication contient plein d'autres choses (je voulais seulement vous mettre l'eau à la bouche) puisqu'elle réunit vingt et un articles bien rangés en quatre parties. J'aimerais au moins en citer les titres, à défaut de pouvoir mentionner tous les auteurs.

Première partie : Astérix autour du monde - Diffusion.

Seconde partie : Autour du monde d'Astérix - Lectures.

Troisième partie : Le tour du monde d'Astérix - Traductions.

Quatrième partie : Les mondes d'Astérix - Adaptations.

C'est la troisième partie qui est la plus étoffée. Tant mieux ! C'est celle qui nous concerne (et nous intéresse) le plus, nous autres traducteurs, et l'on se prend de compassion et d'admiration pour les confrères qui ont dû traduire les aventures du petit Gaulois en arabe, en japonais ou en latin. Cent douze langues en tout, dont beaucoup de langues régionales!

Cette partie me rappelle d'ailleurs les tables rondes organisées dans le cadre des Assises de la traduction littéraire en Arles, qui réunissent plusieurs traducteurs et traductrices d'un même auteur et permettent de se rendre compte, accessoirement, que les problèmes de traduction les plus épineux ne sont pas forcément ceux auxquels on aurait spontanément pensé(2).

Les autres parties du Tour du monde d'Astérix ne manquent pas pour autant d'intérêt pour les traducteurs que nous sommes. Plusieurs articles abordent des questions sociologiques sous

(2) Une année, la table ronde s'est intéressée aux traductions des Exercices de style de Raymond Queneau. Autre gageure! 
un angle plus ou moins théorique, d'autres le phénomène éditorial qu'a été Astérix et ses fortunes diverses selon les pays - succès monumental ou flop - pour des raisons difficiles à prévoir, et parfois même à expliquer après coup.

Mais puisque j'aborde les choses par le petit bout de la lorgnette, je voudrais citer cette réflexion - pas tout à fait anecdotique non plus - de Goscinny :

Je ne peux pas faire autant de calembours que je voudrais, parce qu'il faut tout de même que je pense un peu aux traducteurs étrangers qui s'arrachent les cheveux dès que je fais un calembour (p. 34).

Curieux retour de bâton. Milan Kundera aussi (échaudé sans doute par une mauvaise expérience avec la traduction en français de La Plaisanterie) ne peut s'empêcher d'écrire en pensant aux traducteurs, ou du moins aux problèmes que posera la traduction de ses romans en langue étrangère. Cette prise en compte de la traduction dès le stade de l'écriture n'est-elle pas aussi un phénomène intéressant ? À quand donc une étude sur "l'écriture contrôlée " chez les écrivains?

Mais je m'égare et j'ai épuisé mon temps de parole. Avant d'oublier, voici donc les références de ce magnum opus sur l'indomptable héros national des Français. Je précise que la présentation de l'ouvrage est très soignée, ce qui n'est pas pour déplaire aux professionnels que nous sommes.

Bernard Richet (éd.), Le Tour du monde d'Astérix, Actes du colloque tenu à la Sorbonne les 30 et 31 octobre 2009, Paris, Presses Sorbonne nouvelle, 2011, 320 pages, ISBN 978-287854-514-2. 


\section{EXERCICEPRATIX}

Soit l'expression " je suis médusé " à traduire dans une langue étrangère " $\mathrm{X}$ ".

II serait étonnant de ne pas trouver un mot qui exprime l'idée d'être fasciné, stupéfait, éberlué, pétrifié - à adapter selon le contexte.

Mais, dans la vignette ci-dessous, tirée d'Astérix légionnaire(1), le sens de ce que dit le pirate importe moins que la "référence culturelle iconique " au Radeau de la Méduse, comme nous le rappelle opportunément Nathalie Sinagra(2) dans son analyse de quelques traductions d'Astérix. Dès lors, comment rendre ce "clin d'œil au lecteur " ?

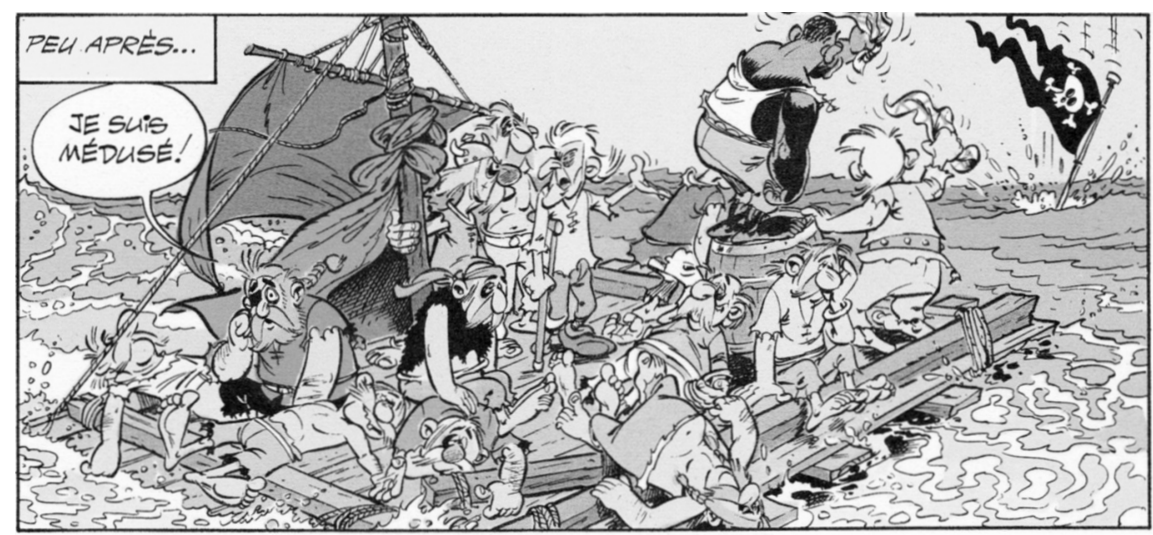

Astérix légionnaire, Goscinny et Uderzo, page 35.

L'auteure nous présente quelques-unes des solutions adoptées.

Le traducteur italien a opté pour un Mi hanno medusato(3), en expliquant en note l'allusion à un tableau au Louvre (sans préciser le nom du peintre). Le mot medusato n'existe pas en italien, mais on voit déjà sur internet plusieurs sites qui expliquent le sens et le contexte de l'expression. Rien n'interdit donc qu'elle entre un jour dans la langue.

Le traducteur espagnol fait dire au personnage Por medusa, qué vida esta!(4), expression peu compréhensible, nous dit l'auteure de l'article, bâtie sur le modèle de Por Dios. (Dans

(1) Astérix légionnaire, texte de Goscinny, dessins d'Uderzo, Dargaud S.A. Éditeur.

(2) Nathalie Sinagra, "Traduire Astérix : atouts et contraintes ", in Bertrand Richet (éd.), Le Tour du monde d'Astérix. Voir recension p. 96.

(3) Goscinny, R. et Uderzo, A., Asterix legionario, trad. Marcello Marchesi, Milan, Mondadori, 1968.

(4) Goscinny, R. et Uderzo, A., Asterix legionario, trad. Jaime Perich, Barcelone, Salvat, 1980. 
ce cas, pourquoi pas une majuscule à Medusa puisque le mot désigne à la fois l'animal visqueux et le personnage mythologique?)

Les traducteurs anglais(5), quant à eux, semblent plus inspirés (ou peut-être leur langue, en l'occurrence, se prête-t-elle plus facilement à des facéties verbales). Ils proposent We've been framed, by Jericho, avec une note en bas de page expliquant que Jericho (Géricault !) est un peintre gaulois. L'exclamation sonne un peu comme By Jove, mais, sans la note, je doute que beaucoup d'anglophones auraient fait le lien avec le peintre. Deuxième jeu de mot : We've been framed, qui veut dire littéralement " nous avons été encadrés " (allusion au cadre du tableau - pour les durs de l'entendement), mais aussi "c'est un coup monté ", " on nous a bien eus".

Cette petite " étude de cas " suscite en moi quelques questions :

1. Est-ce que les traducteurs d'Astérix s'arrachent les cheveux ou est-ce qu'ils s'éclatent?

2. Dans l'exemple du Radeau de la Méduse, quelle est la proportion de lecteurs francophones qui comprennent l'allusion(6) ?

3. Faut-il multiplier les notes explicatives, pour faire œuvre de pédagogie vis-à-vis du lecteur, mais aussi pour montrer qu'on a compris l'allusion même si on n'a pas su la rendre?

4. Y-a-t-il lieu de défendre l'idée d'un travail en binôme dans lequel le traducteur s'appuierait sur un informateur monolingue connaissant bien la langue et la culture d'origine?

5. L'impossibilité de traduire certaines images, notamment entre cultures très éloignées, justifierait-elle le principe d'un remake d'une bande dessinée (comme on le fait au cinéma) ?

6. Pourquoi Lacan est-il si apprécié aux États-Unis alors que la plupart de ses jeux sur les mots sont probablement intraduisibles?

7. Etc., etc.

Finalement, devant beaucoup d'images, sérieuses ou non sérieuses, on a envie de dire, comme Daniel Arasse : "On n'y voit rien(7) ! " Je traduis actuellement un ouvrage sur Le Jardin des délices de Bosch, et je n'arrête pas de me dire : "C'est fou tout ce qu'on ne voit pas!"

(5) Goscinny, R. et Uderzo, A., Asterix the Legionary, trad. Anthea Bell et Derek Hockridge, Leicester, Brockhampton Press, 1970.

(6) Mais même si elle n'est pas comprise, elle est toujours là, en filigrane. En revanche, si elle n'est pas traduite (ou expliquée), elle est totalement inaccessible au lecteur étranger.

(7) Titre d'un ouvrage de l'historien de l'art Daniel Arasse (On n'y voit rien, Descriptions, Paris, Denoël, 2000 pour la première édition, republié en édition de poche dans la collection Folio essais en 2003). 